\title{
Test-retest repeatability of the NX-16: a three-dimensional (3D) body scanner in a male cohort
}

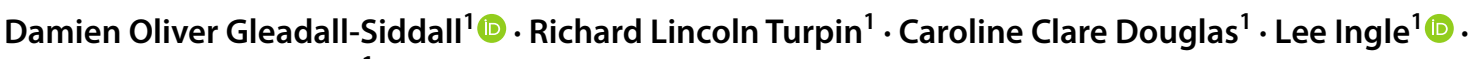 \\ Andrew Thomas Garrett ${ }^{1}$
}

Received: 2 October 2019 / Accepted: 30 November 2019 / Published online: 13 December 2019

(c) The Author(s) 2019

\begin{abstract}
Purpose Whole-body three-dimensional scanning is a tool utilised for the collection of body girths, volume, and surface area measurements. Few studies have investigated the validity and repeatability of this technology. The aim of the present study was to investigate the test retest variability of the NX-16 body scanner (NX-16, TC2, Cary, North Carolina, USA).

Methods Phase one involved the measurement of a mannequin on 300 occasions ( 30 scans over 10 sessions). In phase two, 13 apparently healthy male participants were recruited; each participant was scanned a total of four times (two scans over two sessions). Stature, body mass, and body fat $\%$ were obtained. Fourteen girth measurements were obtained (chest, underbust, stomach, waist, seat, hip, R/L bicep, R/L thigh, R/L mid-thigh, and R/L calf). Coefficient of variation was calculated for measurements obtained.

Results Coefficient of variation for phase one ranged from $0.0 \%$ for the $\mathrm{R}$ calf, to $3.3 \%$ for the $\mathrm{L}$ thigh measurement. For phase two, values were higher, ranging from $0.5 \%$ for calf and chest to $4.6 \%$ for thigh measurements.

Conclusions Test-retest variability of the measurements provided by the NX-16 body scanner varied according to body location. However, variability within measurements was low using a mannequin or human participant. The NX-16 body scanner (TC2, Cary, North Carolina, USA) may be a useful tool for tracking changes in body composition over time during large population studies.
\end{abstract}

Keywords Body scanning $\cdot$ Body composition $\cdot$ Anthropometrics $\cdot$ Circumference measurements

\section{Introduction}

Anthropometry is the ancient practise of measuring body shape and size. Traditional methods involve using a stadiometer, balance scale, or skinfold callipers in order to obtain measures. Advances in technology have enabled researchers and medical professionals alike to gain a deeper insight into the human body; for example, magnetic resonance imaging (MRI), dual-energy X-ray absorptiometry (DEXA), and computed tomography (CT) provide relatively non-invasive, multi-component methods for determining the composition of an organism [1]. However, these imaging techniques (MRI, CT, and DEXA) are not widely available outside of a clinical setting or for repeat measurements due to cost of

Damien Oliver Gleadall-Siddall

d.gleadall-siddall@myacademic.email

1 Department of Sport, Health and Exercise Science, University of Hull, Cottingham Road, Hull HU6 7RX, UK testing, requirement for specialist interpretation of data, and participants' exposure to ionising radiation.

Anthropometric measures are widely reported within the fields of medicine, nutrition, and sports science in order to assess disease risk and monitor health. Stature and body mass are used for the calculation of body mass index (BMI), a measure of obesity, which has links to increased risk of mortality and morbidity [2]. Increased fat mass is associated with chronic disease including type 2 diabetes [3] and can be assessed via bioelectrical impedance analysis [4]. Waistto-hip ratio, also linked with increased disease risk (above a specific threshold), can be obtained through the measurement of body girths using a flexible tape measure [5].

The measurement of circumferences within specific body compartments provides an accessible measure for determining disease risk and tracking the efficacy of a lifestyle intervention [6]. For example, waist-to-hip ratios simply require the use of a tape measure; this measurement is repeatable with a reported coefficient of variation 
$(\mathrm{CV} \%)<3 \%$ requiring minimal training by the investigator [7]. Measuring circumferences of other body compartments including the arms, chest, and legs may also be useful in population-based investigations. However, measuring multiple circumferences in different body compartments can present increased challenges such as an increased time burden, participant discomfort, and ethical issues surrounding the need to remove clothing.

Commercially available devices make it possible to rapidly obtain circumferences from multiple compartments of the human body. Three-dimensional (3D) body scanning employs laser or light sources, which are projected onto the body surface and cameras detect the distortion pattern created [7]. Computer software then renders a 3D model, automatically detecting landmarks whilst determining the magnitude of the body compartment [8]. Body scanning has been used within a healthcare setting [8], research environment $[9,10]$, and within sport [11]. Multiple devices are commercially available including the NX-16 body scanner (NX-16, TC2, Cary, North Carolina, USA).

As with any automated device, the observed value of a measurement can be different from the true value due to measurement error [12]. Many factors affect the difference between the true and observed values, but the two most common are the validity and reliability of a measure [12]. Validity refers to the precision of the measurement, its ability to report the "true" value of the intended marker, whereas reliability or repeatability refers to the random variation/ error in values provided by the same measure re-assessed on multiple occasions. The validity of the NX-16 scanner has been investigated previously comparing values obtained with the criterion method of a flexible tape measure [7]. The NX-16 scanner has good validity for various measurement sites including the thigh, arms, chest, and waist [9, 13]. However, there is limited data available relating to the repeatability of the NX-16 over multiple testing sessions. There is also limited guidance for the calibration of the scanner during repeated usage. Therefore, the aim of the present study was to investigate the test retest repeatability of the $\mathrm{NX}-16$ body scanner in order to inform recommendations for its use.

\section{Methods}

The study received institutional ethical approval from the Sport, Health and Exercise Science Ethics Committee at the University of Hull, and was conducted in accordance with the Declaration of Helsinki [14]. Written informed consent was obtained from each participant prior to their involvement in the study.

This study involved two separate investigations using a computerised 3D body scanner (NX-16, TC2, Cary, North
Carolina, USA); phase one used a mannequin scanned multiple times on multiple occasions. The rationale for the two-phase process including the use of a standardised mannequin was to predominantly limit the sources of measurement error which would be associated with an assessment of human participants only. The practicality of collecting hundreds of individual scans also precludes human-only use as individuals are less likely to be available over multiple testing sessions. Thirty scans per session were chosen as we estimated this to be in excess of the total number of scans an investigator would feasibly complete in a single working day. The total number of scans we estimated would provide enough data to enable a meaningful interpretation of the data and allow practical device recommendations to be developed. Phase two involved a study using human participants who were each scanned four times over two visits. Visits for phase two were scheduled at the same time of day to account for diurnal variation. The same equipment was used for all visits and operated by the same test administrator. Figure 1 provides a visual schematic outlining the study design.

Anthropometric measures were collected as part of the medical screening process and for reporting of participant characteristics. Stature was measured using a wall-mounted stadiometer (Holtain Stadiometer, Holtain Ltd, Crymych, Dyfed), body mass was measured using a balance scale (SECA Balance Scales, Vogel \& Halke, Hamburg Germany), and body fat percentage using bioelectrical impedance (BF-900, Maltron International, Rayleigh, UK); all were measured on arrival at the laboratory.

The NX-16 body scanner has been described previously [13]. The scanner was connected to a desktop PC running Windows XP and utilised specialist software (3D Body Measurement System V2.1, TC2, Cary, North Carolina, USA). External light sources (from outside of the scanner) cause measurement error; therefore, overhead lights were switched off, blinds within the laboratory were closed and both the inner and outer curtains of the NX-16 were drawn.

The NX-16 scanner was calibrated at the start of each testing session. The calibration involves a cylinder of known dimensions (height $151.7 \mathrm{~cm}$; circumference $88.0 \mathrm{~cm}$ ) and 11 "calibration balls" (two hanging wires with five balls and one loose ball). Calibration involved a three-step process. Initially, the cylinder was placed in the centre of the scanner (in between the ten hanging calibration balls) and images were captured. Secondly, the cylinder was removed and the loose calibration ball was placed inside the scanner and a further set of images were captured. Finally, the software uses the captured images to complete the calibration and determine the cylinder circumference. The two sets of hanging calibration balls are required to be perfectly still. The system calibration is considered acceptable if the circumference of the cylinder is within the error margin of $\pm 5 \mathrm{~mm}$ of the true cylinder circumference. The scanner software 


\section{Phase 1}
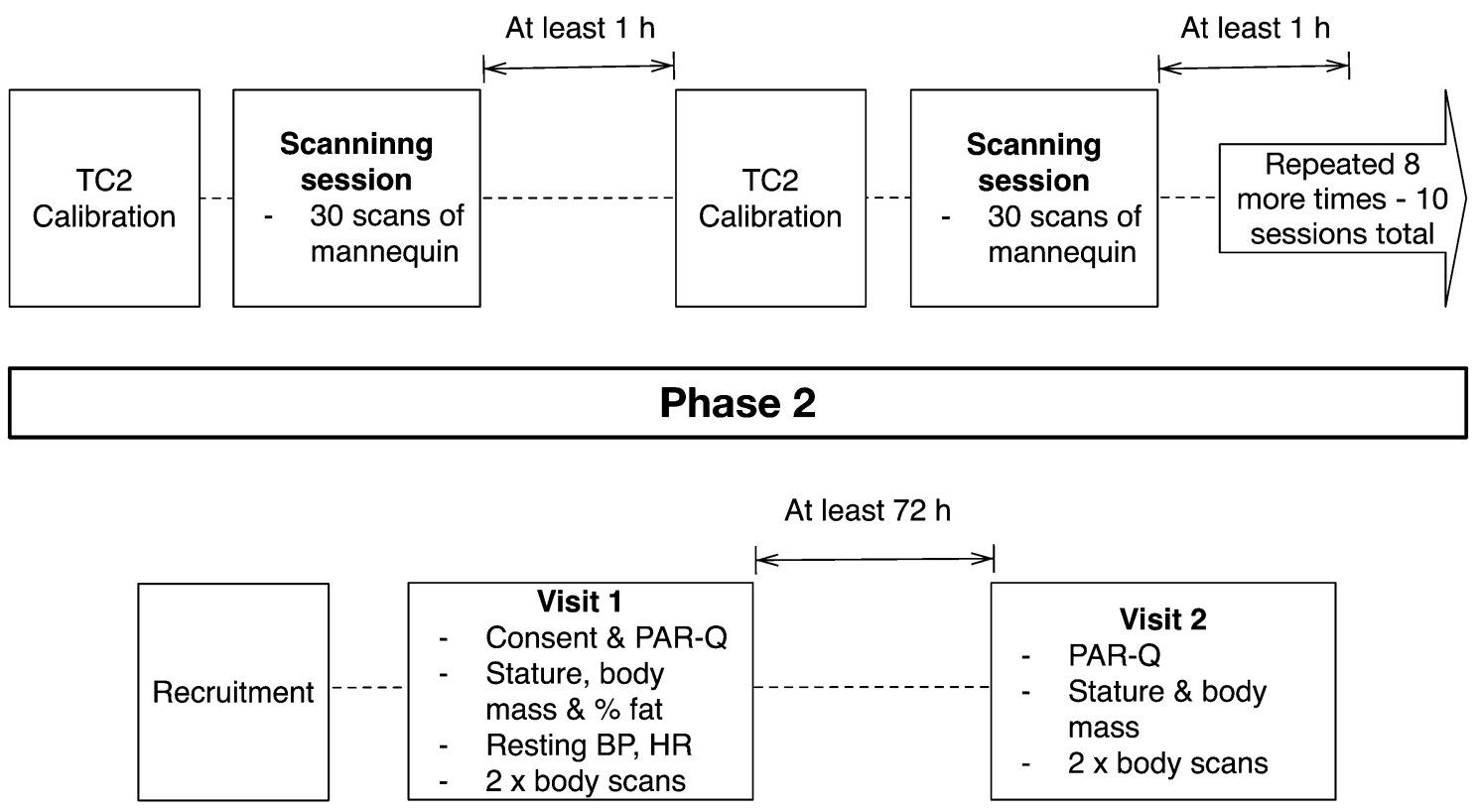

Fig. 1 Visual schematic outlining the study design. Phase 1 relates to the repeat measurement of a male mannequin. Phase 2 involved the measurement of human participants on two separate occasions

allows for the extraction of custom measurement. Measurement locations are shown in Fig. 2.

For phase one, in order to assess the repeatability of the NX-16 scanner, a male mannequin with an athletic build was scanned on 300 occasions (10 sessions each with 30 scans). A calibration was completed before each session. Following successful calibration, the mannequin was placed in the centre of the scanner with the inner and outer curtains drawn to prevent light leaks. The system calibration was verified following the scanning session by scanning the reference cylinder.

For phase two, apparently healthy male participants were recruited. Participants attended the laboratory on two occasions. Anthropometric measurements were collected on arrival. Two body scans were then obtained for each participant. The NX-16 scanner was calibrated at around 08:30 $\mathrm{h}$ and again at 12:00 $\mathrm{h}$ each day. Participants were asked to undress behind the outer privacy curtain, removing all jewellery and clothing, except underwear. Entering the scanner, participants closed the inner curtain, stood in the centre of the scanner with their feet around hip width apart; participants were instructed to stand upright, looking straight ahead with their chin slightly raised. During the scanning process, participants were asked to remain still. Immediately following scanning, participants remained in the scanner until the data were processed. A second scan was immediately completed using the same protocol. Participants wore white or light-coloured underwear for all scans.

For phase one, averages for each measurement location were obtained for the first and second and the third and fourth scans of each session. For phase two, measurements from each visit were averaged. Data were processed using a bespoke spreadsheet designed for variability analysis [http://www.sportsci.org/resource/stats/relycalc.html] [15]. Results are presented as mean \pm standard deviation (SD) unless otherwise stated. Test-retest variability was reported as the typical error of the coefficient of variation $(\mathrm{CV} \%)$ with $90 \%$ confidence intervals (CI). The benefits of using typical error and the statistical methods employed have been outlined previously [12].

\section{Results}

Three-hundred scans of the mannequin were obtained for phase one. Thirteen male participants (mean \pm SD: stature $180.1 \pm 9.2 \mathrm{~cm}$, body mass $82.0 \pm 9.9 \mathrm{~kg}$, BMI $25.4 \pm 3.3 \mathrm{~kg} \mathrm{~m}^{2}$, and body fat $18.4 \pm 8.0 \%$ ) were recruited for phase two of the study. All participants completed both visit one and visit two ( $6 \pm 4$ days between trials). No adverse events were reported throughout the study. During 


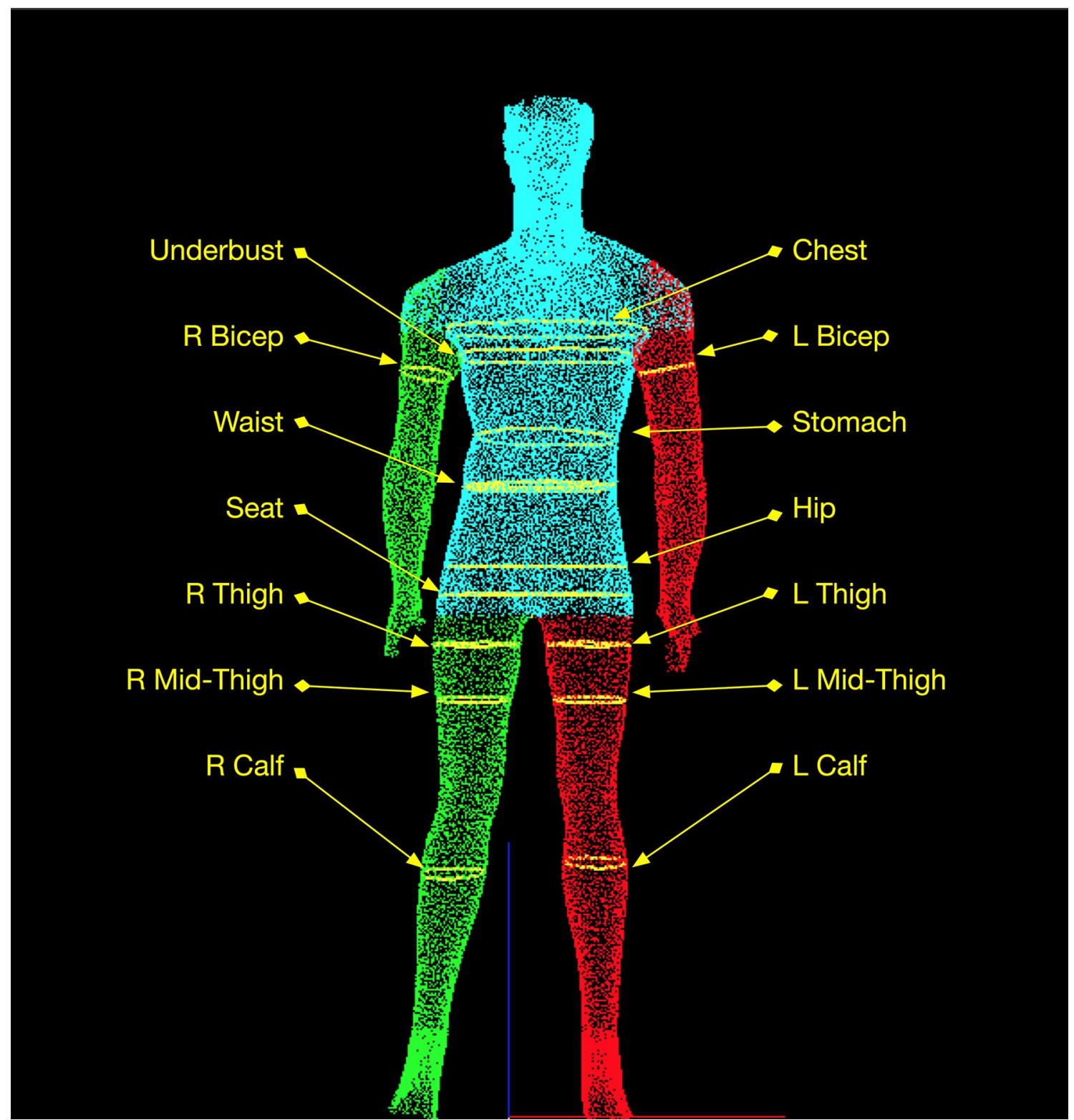

Fig. 2 Screenshot from the NX-16 software identifying the measurement sites used for the study

phase one, time between the final scan of a session and the calibration for the next session was $105 \pm 29 \mathrm{~min}$.

Repeatability data for both phases of the study are presented in Table 1. Measurements of human participants showed greater variability compared to a mannequin (highest CV\% for phase one 3.3\%; highest CV\% for phase two $4.6 \%$ ). The variability when scanning human participants ranged from 0.5 to $4.6 \%$ depending on the measurement location. Data from each of the 300 scans of phase one are provided in Figs. 3 and 4. Data are freely available online in an effort to support research transparency and the open science movement [https://osf.io/y7qk2/] [16].

\section{Discussion}

The aim of the present study was to assess the test-retest repeatability of the NX-16 3D body scanner. We found that the test-retest variability of the measurements provided by the NX-16 body scanner varied according to body location. Indeed, variability within measurements was low using a mannequin or human participant. Our findings demonstrate a $\mathrm{CV} \%$ ranging from 0.5 to $4.6 \%$ dependent upon measurement location. Due to the inherent variability in $\mathrm{CV} \%$ values in different body compartments, it would seem logical therefore for researchers to focus on specific 
Table 1 Calculated CV and 90\% CI for each measurement site for phase one and phase two

\begin{tabular}{lllllll}
\hline Measurement & $\begin{array}{l}\text { Phase 1 } \\
\text { CV }(\%)\end{array}$ & Lower 90\% CI & Upper 90\% CI & $\begin{array}{l}\text { Phase 2 } \\
\text { CV }(\%)\end{array}$ & Lower 90\% CI & Upper 90\% CI \\
\hline Chest & 0.2 & 0.2 & 0.4 & 0.5 & 0.4 & 0.8 \\
Underbust & 0.9 & 0.7 & 1.5 & 4.4 & 3.3 & 6.8 \\
Stomach & 0.1 & 0.1 & 0.2 & 2.4 & 1.8 & 3.6 \\
Waist & 0.3 & 0.2 & 0.5 & 0.9 & 0.7 & 1.4 \\
Seat & 0.8 & 0.6 & 1.3 & 1.1 & 0.8 & 1.6 \\
Hip & 0.7 & 0.5 & 1.2 & 0.6 & 0.4 & 0.9 \\
R bicep & 2.0 & 1.5 & 3.4 & 2.0 & 1.5 & 3.1 \\
L bicep & 1.3 & 1.0 & 2.2 & 1.7 & 1.2 & 2.5 \\
R thigh & 3.3 & 2.4 & 5.4 & 4.6 & 3.5 & 7.1 \\
L thigh & 3.3 & 2.4 & 5.5 & 4.6 & 3.5 & 7.1 \\
R mid-thigh & 0.8 & 0.6 & 1.3 & 2.6 & 1.9 & 3.9 \\
L mid-thigh & 1.0 & 0.7 & 1.6 & 2,5 & 1.9 & 3.9 \\
R calf & 0.0 & 0.0 & 0.1 & 0.5 & 0.4 & 0.7 \\
L calf & 0.1 & 0.0 & 0.1 & 0.5 & 0.4 & 0.8
\end{tabular}

landmarks to minimise measurement error (e.g. in chest and calf sites) if this is feasible.

The body compartments showing the lowest variability were the chest $(0.5 \%)$, waist $(0.9 \%)$, seat $(1.1 \%)$, hip $(0.6 \%)$, and calf $(0.5 \%)$ measurements sites. The stomach $(2.4 \%)$, bicep (2.0\% and $1.7 \%)$, and mid-thigh (2.6\% and $2.5 \%)$ measurements had a slightly higher variability, whereas the underbust (4.4\%) and both thigh measurements (4.6\%) showed the largest variability when human participants were scanned. Whilst no clear thresholds are available, a reduction of $5 \%$ in waist circumference is suggested to offer a clinically important difference — changes in waist circumference above 5\% likely promote improvements in health [17]. Assessment of measurement error in clinical practice revealed values ranging from 1.4 to $15.0 \mathrm{~cm}$ for manual waist circumference measurements [18]. Our data suggest the NX-16 has a lower $\mathrm{CV} \%$ than the minimal clinically important difference, which would enable researchers and clinicians to detect clinically meaningful changes in waist circumferences.

A number of factors may contribute to the variability in circumference measurements obtained by the scanner. Firstly, the exact location of a measurement may vary from scan to scan; the scanning software uses pre-programmed algorithms to determine the measurement sites. When repeated measurements are taken over multiple trials, small changes in the participant's posture will likely occur, which may impact where the software takes a measurement, resulting in a difference in values, as seen when children were measured using the scanner [19]. Secondly, the small changes in posture may also result in a physical change in how subcutaneous tissue is distributed, therefore changing the girth of a particular area. These two factors will likely compound the variability observed. Finally, the system calibration will also act as another source of error; during phase one, we verified the system calibration at the end of each scanning session with only two of ten calibrations still valid after 30 scans. We believe these are the most likely sources of variability. Practical methodological recommendations will be discussed in an effort to limit these sources of error.

To date, few studies have investigated the test-retest repeatability of the NX-16 scanner, previous relevant studies are summarised in Table 2. Studies that have directly investigated the NX-16 scanner have demonstrated good agreement between the scanner and traditional methods [9, 13]. For example, mean differences in circumference measurements were $<1.0 \mathrm{~cm}$ for multiple measurement locations (arms, thigh, and calf) when compared with traditional tape measure methods [13]. Investigation of similar devices also reports relatively low differences between automated and manual measures (waist $0.64 \mathrm{~cm}$ ) [7]. For weight management, changes of $5 \%$ would be considered the minimal clinically important difference [17]. Measurements from the present study have a CV\% below the proposed 5\% clinically significant change, suggesting that researchers and clinicians can use the NX-16 scanner to detect clinically meaningful changes in circumference measures.

In addition to assessing the repeatability of the NX-16, the aim of the present study was to offer evidence-based recommendations for using the scanning device. Firstly, the system calibration is a straightforward process but the process requires that the hanging calibration balls are perfectly still, and so, this makes it difficult to recalibrate the scanner between scans. For our study, there was an average of 105 min between the end of a scanning session and the next system calibration. Anecdotally, we found that the calibration balls needed to be allowed to hang for a minimum of $60 \mathrm{~min}$ - any less, and the two strings of balls would still be moving slightly and prevent calibration. In phase one, after 30 scans each session, the 
Fig. 3 Raw data, mean, and SD from each scan of phase one. Figure includes data for the arm, leg, and calf measurement sites

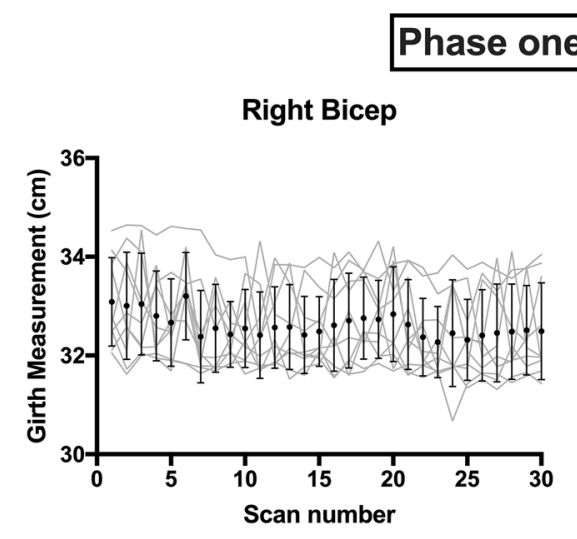

Right Thigh

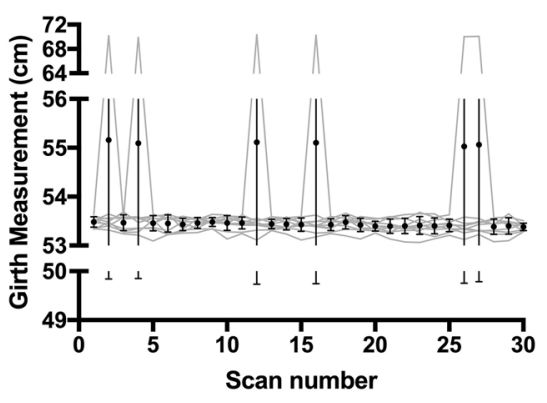

Right Mid-Thigh

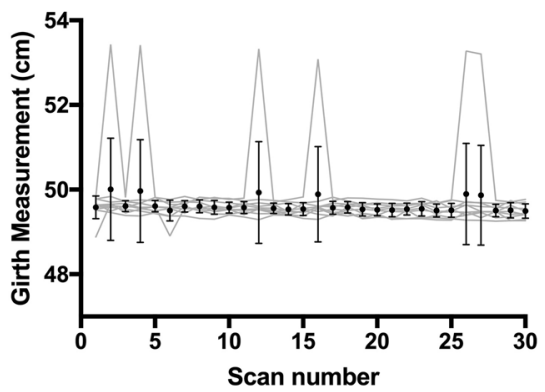

Right Calf

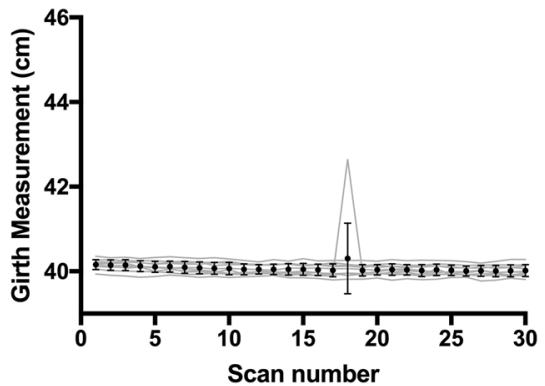

Left Bicep

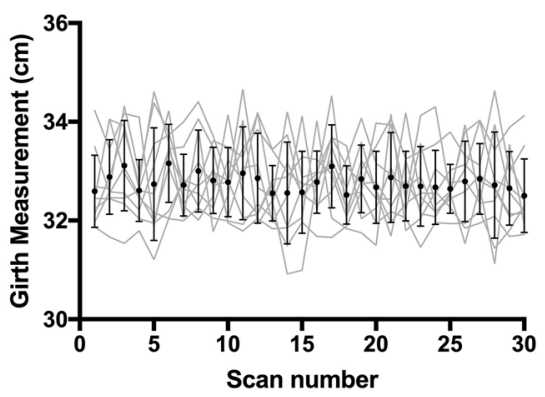

Left Thigh

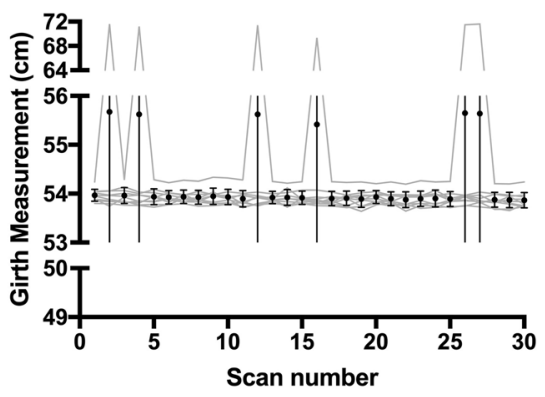

Left Mid-Thigh

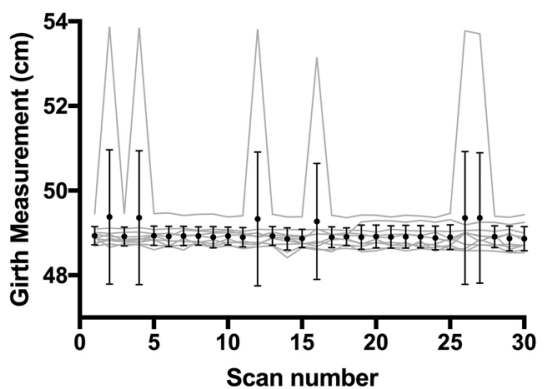

Left Calf

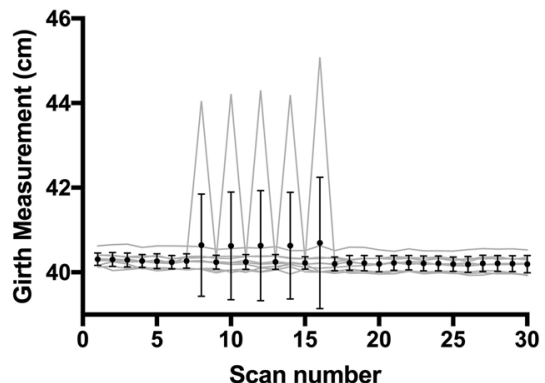

calibration was valid for only two of the ten sessions suggesting that calibration should be completed at least every 30 scans, if not earlier; visually examining the data in Figs. 3 and 4, it appears that the standard deviations of measurements increase after around 20 scans. Therefore, we would recommend that a system calibration is completed after $\sim 20$ scans and that users should verify the system calibration by using the in-built function that scans the reference cylinder-similar to the calibration process. Whilst the system calibration is an important consideration, error from other factors can also be controlled. 


\section{Phase one - Mannequin Upper Body}
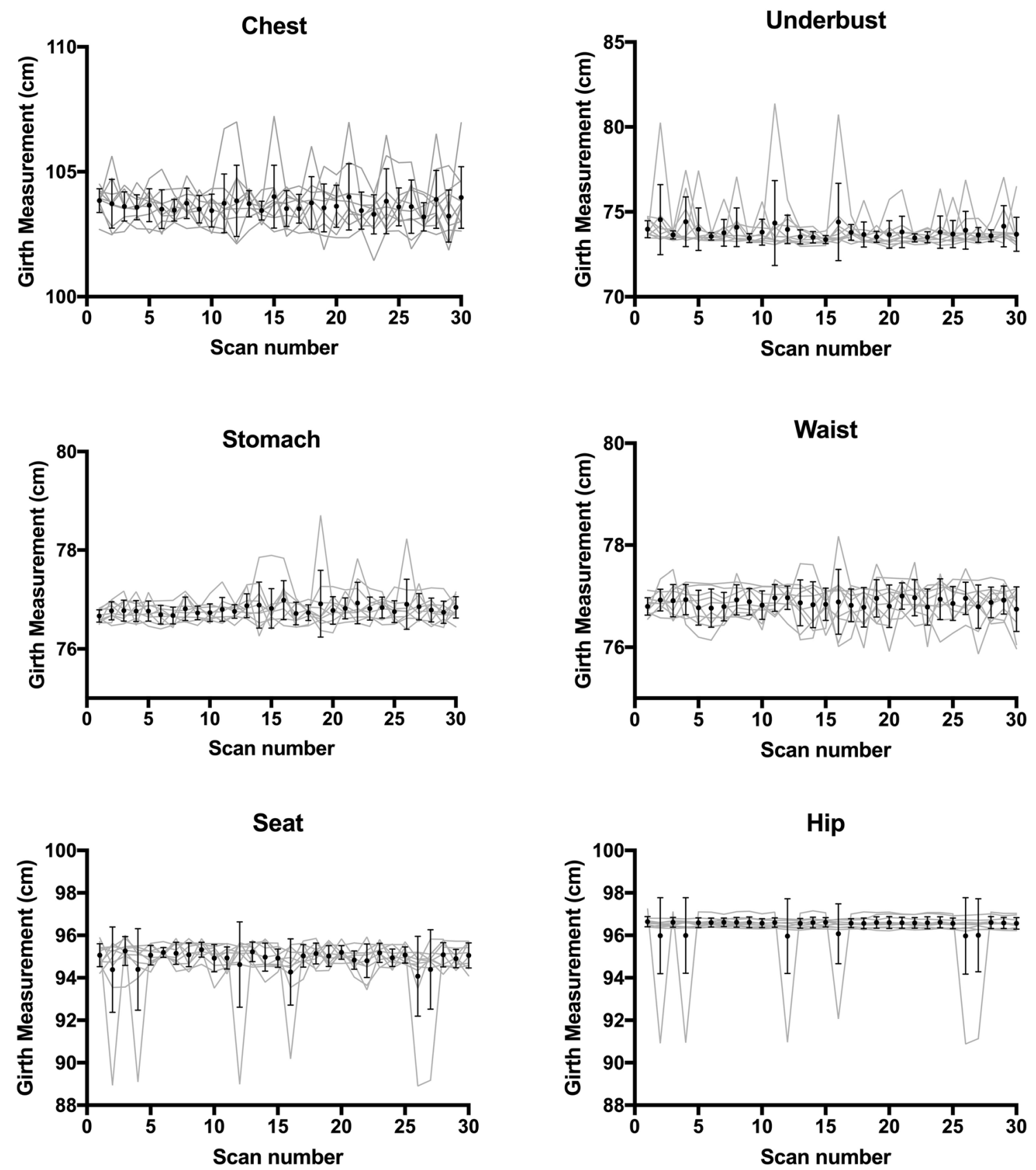

Fig. 4 Raw data, mean, and SD from each scan of phase one. Figure includes data for the chest, underbust, stomach, waist, seat, and hip measurement sites

Other factors may contribute to increased variability. Researchers have stressed the importance of participants' wearing form-fitting underwear during scanning and the need to prevent external sources of light from entering the scanner [13]. Light leaks can result in artefact within the body model; therefore, we would recommend that users ensure both curtains of the scanner are closed, overhead lights dimmed/switched off, and room blinds/curtains are closed. Our results also demonstrate that differences in the precise measurement location can occur between scans. This may be due to minute variations in posture between scans; we would recommend that participants are provided with adequate instructions before scanning, that users follow the positioning outlined within the methods section 


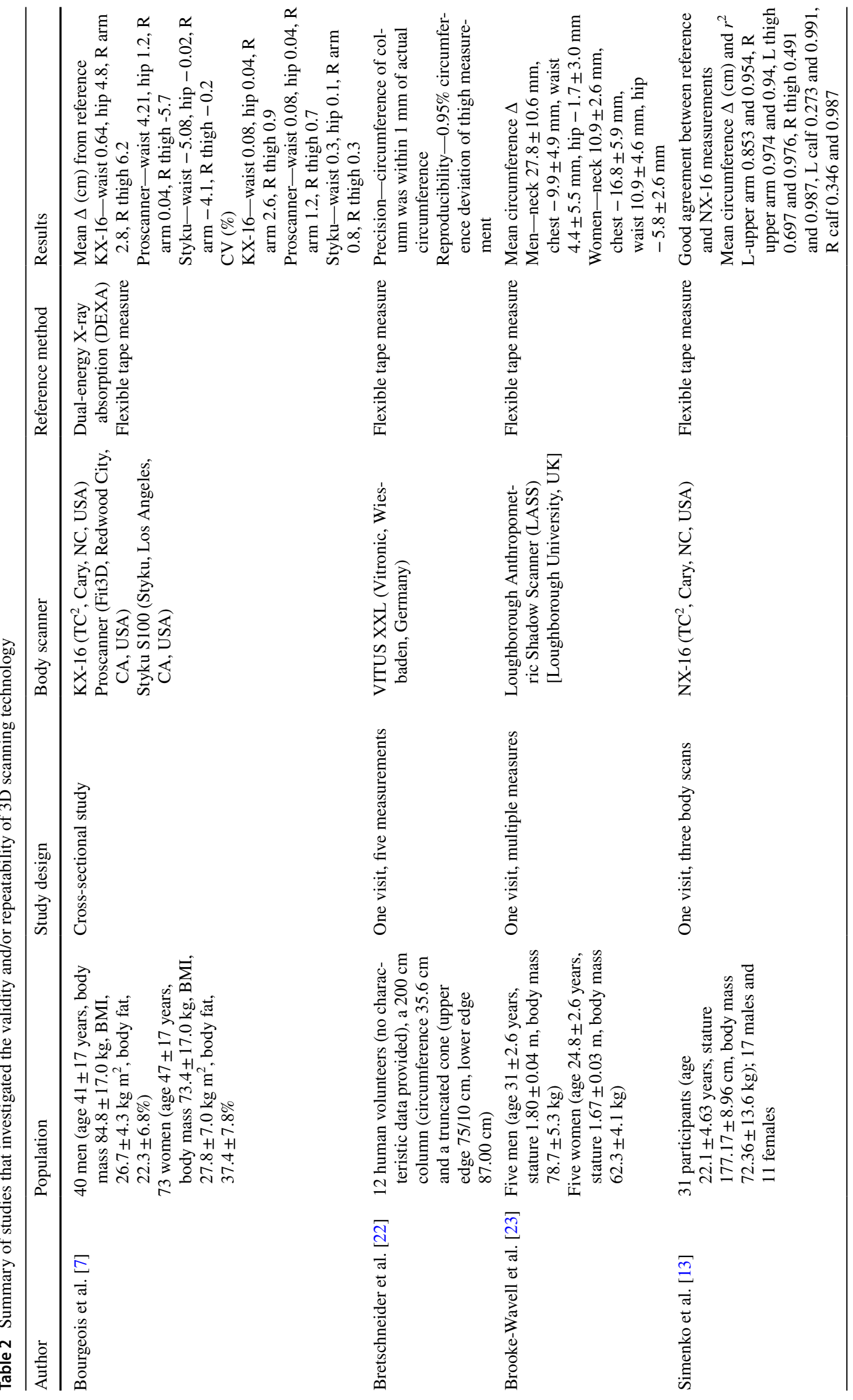




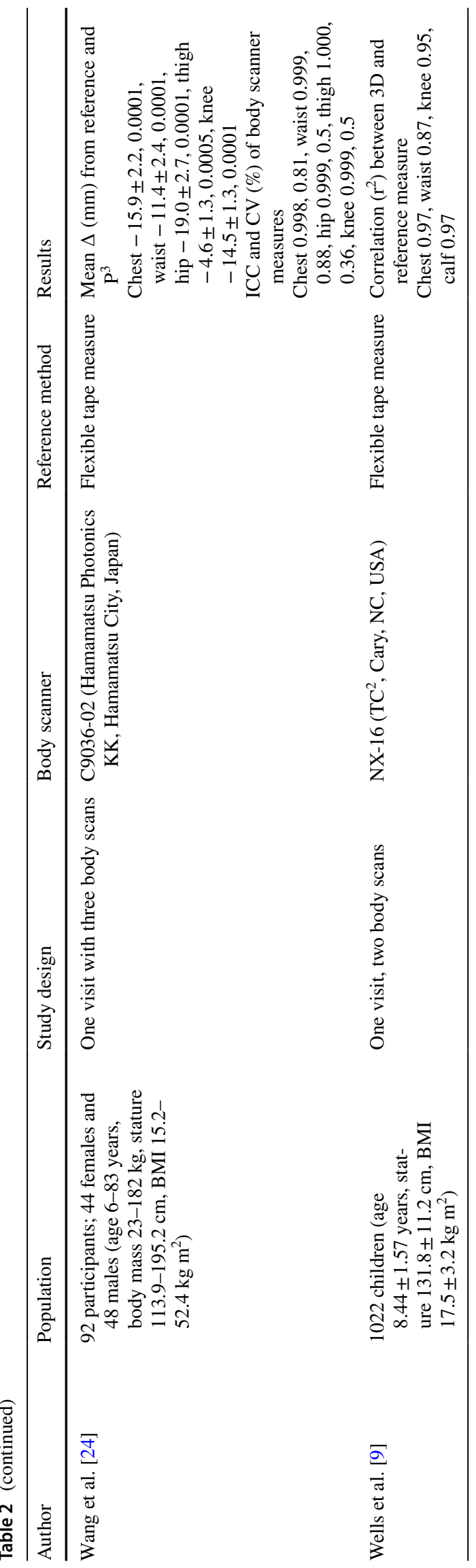

and attempt to ensure consistency throughout. Underwear worn by participants should be form-fitting and also white or lighted coloured to ensure a successful scan; anecdotally, we noticed that dark-coloured underwear prevented the scanner from rending the covered section of a participant, resulting in measurement failure. Users should capture duplicate scans at a minimum and report the mean values. Data from phase one of the study demonstrated that intermittent random errors can occur (Figs. 3 and 4), which can be easily excluded and another scan completed.

3D body scanning technology has advanced over time; various models are commercially available, and the technology offers benefits over traditional methods such as the flexible tape measure. Body scanners such as the NX-16 can scan a participant and generate a whole-body model in less than $60 \mathrm{~s}$, which would take a considerable amount of time if the traditional tape measure method was used. The technology also allows retrospective analysis of scans, extraction of additional measurements at a later date, and the calculation of variables such as surface area and body volume. New operators of the scanner can be easily trained compared to someone learning to use skinfold callipers; for example, someone wishing to become ISAK accredited would need considerable training and practice in order to become certified [20]. Participants may also feel uncomfortable with the traditional calliper or tape measure methods as there is a need to remove clothing in front of the test administrator; whilst participants also need to undress using the body scanner, they do so behind a privacy curtain, out of sight from others, and in a darkened environment.

Future research may wish to explore the technology further. Firstly, different measurement sites may be of interest, such as the calculation of overall body surface area or volume. These measurements, such as surface area, may have clinical importance or prognostic value. Secondly, the present study investigated only adult males, and therefore, the analysis of females and other age groups would expand the understanding of the technology allowing normative data for males and females within age strata to be developed. Further, the development of minimal clinically important difference thresholds could also be developed. Finally, the investigation of skin pigmentation on the validity of the technology would also be useful; research of similar technology utilising light sources, such as photoplethysmography (PPG), has shown some additional variability for participants with darker skin pigmentation [21].

\section{Conclusions}

In conclusion, the test-retest variability of the measurements provided by the NX-16 body scanner varied according to body location. However, variability within measurements 
was low using a mannequin or human participant. Data suggest that users should perform a new calibration at least every 30 scans, ensure participants hold a consistent posture during repeat scans, should wear white or light-coloured form-fitting underwear, and light leaks from external sources are prevented. The NX-16 body scanner (TC2, Cary, North Carolina, USA) may be a useful tool for tracking changes in body composition over time during large population studies.

Acknowledgements The authors would like to express their gratitude to the participants who volunteered for the study - their time and effort are appreciated.

\section{Compliance with ethical standards}

Conflict of interest The authors declare that no external funding was associated with this study. We can confirm that our original findings are not under review elsewhere. The authors declare no conflicts of interest in relation to this study.

Ethical approval All procedures performed in studies involving human participants were in accordance with the ethical standards of the institutional and/or national research committee (Department of Sport, Health and Exercise Science at The University of Hull) and with the 1964 Helsinki declaration and its later amendments or comparable ethical standards.

Informed consent Written informed consent was obtained from each participant prior to their involvement in the study.

Open Access This article is licensed under a Creative Commons Attribution 4.0 International License, which permits use, sharing, adaptation, distribution and reproduction in any medium or format, as long as you give appropriate credit to the original author(s) and the source, provide a link to the Creative Commons licence, and indicate if changes were made. The images or other third party material in this article are included in the article's Creative Commons licence, unless indicated otherwise in a credit line to the material. If material is not included in the article's Creative Commons licence and your intended use is not permitted by statutory regulation or exceeds the permitted use, you will need to obtain permission directly from the copyright holder. To view a copy of this licence, visit http://creativecommons.org/licenses/by/4.0/.

\section{References}

1. Ackland TR et al (2012) Current status of body composition assessment in sport review and position statement on behalf of the ad hoc research working group on body composition health and performance, under the auspices of the I.O.C medical commission. Sports Med 42(3):227-249

2. Must A et al (1992) Long-term morbidity and mortality of overweight adolescents - a follow-up of the harvard growth study of 1922 to 1935. N Engl J Med 327(19):1350-1355

3. Brancati FL et al (1999) Body weight patterns from 20 to 49 years of age and subsequent risk for diabetes mellitus- the Johns Hopkins precursors study. Arch Intern Med 159(9):957-963

4. Bosy-Westphal A et al (2013) What makes a BIA equation unique? Validity of eight-electrode multifrequency BIA to estimate body composition in a healthy adult population. Eur J Clin Nutr 67:S14-S21
5. Janiszewski PM, Janssen I, Ross R (2007) Does waist circumference predict diabetes and cardiovascular disease beyond commonly evaluated cardiometabolic risk factors? Diabetes Care 30(12):3105-3109

6. Madden AM, Smith S (2016) Body composition and morphological assessment of nutritional status in adults: a review of anthropometric variables. J Hum Nutr Diet 29(1):7-25

7. Bourgeois B et al (2017) Clinically applicable optical imaging technology for body size and shape analysis: comparison of systems differing in design. Eur J Clin Nutr 71(11):1329-1335

8. Treleaven P, Wells J (2007) 3D body scanning and healthcare applications. Computer 40(7):28

9. Wells JCK et al (2015) Acceptability, precision and accuracy of 3D photonic scanning for measurement of body shape in a multiethnic sample of children aged 5-11 years: the SLIC study. PLoS One 10(4):15

10. Gropper SS et al (2012) Changes in body weight, composition, and shape: a 4-year study of college students. Appl Physiol Nutr Metab 37(6):1118-1123

11. Schranz N et al (2010) Three-dimensional anthropometric analysis: differences between elite Australian rowers and the general population. J Sports Sci 28(5):459-469

12. Hopkins WG (2000) Measures of reliability in sports medicine and science. Sports Med 30(1):1-15

13. Simenko J, Cuk I (2016) Reliability and validity of NX-16 3D body scanner. Int J Morphol 34(4):1506-1514

14. World Medical Association (2018) W. WMA Declaration of Helsinki-ethical principles for medical research involving human subjects. https://www.wma.net/policies-post/wma-declaration-ofhelsinki-ethical-principles-for-medical-research-involving-human -subjects/. Accessed 15 July 2018

15. Hopkins WG (2000) Calculations for reliability (Excel spreadsheet). A new view of statistics $2000 \mathrm{http}: / / \mathrm{www}$.sportsci.org/ resource/stats/relycalc.html. Accessed 15 July 2018

16. Gleadall-Siddall DO et al. (2019) Test-retest repeatability of the $\mathrm{NX}-16$, a three-dimensional (3D) body scanner in a male cohort. https://osf.io/y7qk2/. Accessed 24 Aug 2019

17. Stevens $J$ et al (2006) The definition of weight maintenance. Int J Obes 30(3):391-399

18. Verweij LM et al (2013) Measurement error of waist circumference: gaps in knowledge. Public Health Nutr 16(2):281-288

19. Wells JCK, Treleaven P, Cole TJ (2007) BMI compared with 3-dimensional body shape: the UK National Sizing Survey. Am J Clin Nutr 85(2):419-425

20. Stewart AD et al (2011) International Standards for Anthropometric Assessment. International Society for the Advancement of Kinanthropometry, London

21. Shcherbina A et al (2017) Accuracy in wrist-worn, sensor-based measurements of heart rate and energy expenditure in a diverse cohort. J Personal Med 7(2):12

22. Bretschneider T et al (2009) Validation of the body scanner as a measuring tool for a rapid quantification of body shape. Skin Res Technol 15(3):364-369

23. Brookewavell K, Jones PRM, West GM (1994) Reliability and repeatability of 3-D body scanner (LASS) measurements compared to anthropometry. Ann Hum Biol 21(6):571-577

24. Wang J et al (2006) Validation of a 3-dimensional photonic scanner for the measurement of body volumes, dimensions, and percentage body fat. Am J Clin Nutr 83(4):809-816

Publisher's Note Springer Nature remains neutral with regard to jurisdictional claims in published maps and institutional affiliations. 